\title{
Fauna helmintológica de bovinos da região de Jaboticabal, Estado de São Paulo, Brasil
}

\author{
Helminth parasites of cattle from Jaboticabal, São Paulo State, Brazil
}

\author{
Fernando de Almeida Borges ${ }^{1,2}$; Daniela Miyasaka da Silveira ${ }^{1,2}$; Erika Barbosa Neves \\ Graminha $^{1,2}$; Karina Carrão Castagnolli, ${ }^{1,2}$; Vando Edésio Soares ${ }^{1,2}$; Adjair Antônio do Nascimento'; \\ Alvimar José da Costa ${ }^{1}$
}

\begin{abstract}
Resumo: $O$ estudo tem como objetivo avaliar a carga parasitária (helmintos) de bovinos da região de Jaboticabal, Estado de São Paulo. Para tanto, foram utilizados 42 bovinos naturalmente infectados, machos, mestiços (taurinos $x$ zebuínos), com idade variando entre 8 e 14 meses. Os resultados necroscópicos revelaram a presença de 17 espécies de helmintos, com a seguinte prevalência e a média da intensidade de infecção: Haemonchus placei $97,62 \%(1961,81)$; Cooperia punctata 92,86\% (8109,45); Oesophagostomum radiatum 73,81\% (217,38); Trichuris discolor 38,19\% $(25,48)$; Trichostrongylus axei 26,19\% (122,05); H. simillis 21,43\% (37, 45); C. pectinata 19,05\% (98,6); Bunostomum phlebotomum 16,66\% (8,95); Dictyocaulus viviparus 16,66\% (3,62); C. spatulata14,29\% (109,95); Capillaria bovis 11,90\% (5,48); Ostertagia ostertagi $7,14 \%(0,86)$; O. Iyrata 4,76\% (0,17); Eurytrema coelomaticum 4,76\% (2,14); Moniezia benedeni, $4.76 \%(0,05)$; T. colubriformis 2,38\% $(0,48)$; Strongyloides papillosus $2,38 \%(0,25)$. A carga parasitária média foi de 10804 helmintos por animal. Haemonchus e Cooperia corresponderam, respectivamente, a 18,50\% e 77,91\% da carga parasitária média. Dos 42 bovinos necropsiados $71,43 \%$ estavam infectados por três a cinco espécies de helmintos. Apenas 2,38\% dos hospedeiros mostravam-se parasitados por 10 espécies diferentes de helmintos.
\end{abstract}

Palavras-chave: prevalência, helmintos, bovinos.

Abstract: This paper deals with the study of parasites in cattle in the region of Jaboticabal, São Paulo. For this, 42 holstein-zebu mixed breeding animals, 8 to 14 month old, were necropsied. At necropsy 17 species of helminth were collected. The prevalence and intensity of infection were as follow: Haemonchus placei 97,62\% (1961,81); Cooperia punctata $92,86 \%(8109,45)$; Oesophagostomum radiatum $73,81 \%(217,38)$; Trichuris discolor $38,19 \%(25,48)$; Trichostrongylus axei 26,19\% (122,05); H. simillis 21,43\% (37, 45); C. pectinata 19,05\% (198,6); Bunostomum phlebotomum 16,66\% (8,95); Dictyocaulus viviparus 16,66\% (3,62); C. spatulata14,29\% (109,95); Capillaria bovis $11,90 \%$ $(5,48)$; Ostertagia ostertagi 7,14\% (0,86); O. Iyrata 4,76\% (0,17); Eurytrema coelomaticum 4,76\% (2,14); Moniezia benedeni, $4.76 \%(0,05) ;$ T. colubriformis $2,38 \%(0,48)$; Strongyloides papillosus $2,38 \%(0,25)$. Each calf had an average worm burden of 10804 helminths Haemonchus (18,5\%) and Cooperia $(77,91 \%)$ were the most frequently observed helminths. Mixed infections were commonly observed, $71,43 \%$ of the animals were parasitized with three to five different species of helminths. Only $2,38 \%$ of the calves were parasitized with ten different species of helminths.

Key words: prevalence, helminths, cattle.

\section{Introdução}

Os helmintos são responsáveis por grandes perdas econômicas na produção animal, associadas principalmente à inapetência, menor ganho em peso, pobre performance reprodutiva, baixa produção de leite, entre outros.

Os sistemas de criação de bovinos no Brasil especializam-se cada vez mais, procurando ser economicamente viáveis e competitivos com outras atividades. Para atender tais objetivos, uma das medidas de manejo utilizada é a intensificação do uso das pastagens, com aumento da relação unidade animal/área. No entanto, essa condição propicia aos animais maior infecção parasitária, o que acarreta baixo índice de crescimento e aumento da taxa de mortalidade, como já observado no sul do país, onde atinge 10\% (BIANCHIN, 1996). Desta forma, no calendário sanitário de uma propriedade devem estar inclusas aplicações de anti-helmínticos que, utilizados de maneira estratégica, garantem maior produtividade e retorno econômico (FURLONG et al., 1993). Entretanto, esse aspecto deve ser embasado no conhecimento das espécies de helmintos presentes nos bovinos da região, assim como sua epidemiologia.

Com este objetivo, estudos para estabelecer os indicadores de infecções foram realizados na região sudeste por Costa et al. (1970), Costa et al.(1973), Nogueira et al. (1976), Duarte et al.(1982), Guimarães et al.(1983), Oliveira e Matsumoto (1985), Oliveira (1988), Santana et al.(1989), Guimarães et al. (1996), Landim et al. (2001); na região centro-oeste, por Grisi e Nuernberg (1971), Carneiro e Freitas (1977), Melo e Ribeiro (1977), Costa et al. (1979), Catto e Ueno (1981), Bianchin et al. (1996); na região sul por Rassier (1975), Ramos (1983), Santos et al.(1994), Pinheiro (1996) ; na região nordeste por Athayde et al.(1999a e b) e Girão et al.(1999).

\footnotetext{
${ }^{1}$ CPPAR-Centro de Pesquisas em Sanidade Animal, FCAV/UNESP, Campus de Jaboticabal.

${ }^{2}$ Aluno de pós-graduação, FCAV/UNESP, Jaboticabal.
} 
Tendo em vista a necessidade de conhecimento da fauna de helmintos de cada região, o objetivo do presente trabalho é caracterizar a prevalência e a intensidade de parasitismo dos diferentes gêneros de helmintos em bovinos na região de Jaboticabal, Estado de São Paulo.

\section{Material e Métodos}

A pesquisa foi realizada no período de 1999 a 2001 , empregando 42 bovinos pertencentes a diferentes propriedades rurais da região de Jaboticabal. O município está situado no Estado de São Paulo, a 354 km da capital, na posição de $21^{\circ} 16^{\prime}$ latitude sul e $48^{\circ} 19^{\prime \prime}$ de longitude oeste e em $575 \mathrm{~m}$ de altitude.

Os bovinos - machos mestiços holandês $x$ zebu, com idade de 8 a 14 meses - apresentavam-se em razoável estado nutricional e naturalmente infectados por helmintos gastrintestinais. Para sua avaliação e seleção, realizaram-se contagens de ovos por grama de fezes (GORDON e WHITLOCK, 1939 modificado por WHITLOCK, 1948), sendo considerados para o estudo aqueles com contagens de ovos por grama acima de 500 .

Após um período de adaptação de 14 dias em baias individuais do setor II de bovinos, do "Centro de Pesquisas em Sanidade Animal" (CPPAR/FCAVJ/UNESP), onde recebiam ração comercial e silagem de milho, além de água "ad libitum", os animais foram, em seqüência, sacrificados com procedimentos éticos, utilizando-se anestésicos conforme as normas regulamentares recomendadas para pesquisa científica.

Nas necropsias, para recuperação do material, empregou-se a metodologia parasitológica segundo Jacobs et al. (1994). Todo conteúdo e raspado de cada segmento anatômico foram colocados sobre um tamis (tyler 48, abertura 0,297 mm) e lavado. Do conteúdo total de cada segmento, após homogeneização, retirouse uma alíquota de $10 \%$ para exame e estimativa da carga parasitária de helmintos. A parte sólida foi fixada em solução de formol a $5 \%$ aquecido. Também foram examinados fígado, pulmão, rins e coração.

A colheita e contagem genérica dos parasitos, presentes em cada alíquota, foram efetuadas em microscópio estereoscópio (lupa) e a identificação de espécie realizada por meio de microscopia óptica segundo Levine (1968) e Costa (1982).

Os indicadores de infecção foram calculados segundo Margolis et al. (1982).

\section{Resultados e Discussão}

A prevalência, intensidade média e amplitude total de variação de infecção pelas espécies de helmintos, diagnosticadas nos bovinos, encontram-se registradas na Tabela 1. A distribuição dos casos de parasitismo de acordo com os intervalos de classes é apresentada na Tabela 2. A Tabela 3 demonstra a distribuição percentual dos casos de infecções registradas conforme o número de espécies de helmintos presentes em um mesmo hospedeiro.

Os resultados necroscópicos revelam a presença de 17 espécies com carga parasitária global média de 10804 helmintos. A espécie com maior prevalência foi Haemonchus placei (97,62\%), seguido por Cooperia punctata $(92,86 \%)$. Este resultado apresenta-se divergente aos encontrados por autores no Estado de São Paulo (NOGUEIRA et al., 1976; OLIVEIRA e MATSUMOTO, 1985; OLIVEIRA, 1988; SANTANA et al., 1989; LANDIM et al., 2001) e demais regiões do país (COSTA et al., 1970; GRISI e NUERNBERG, 1971; COSTA et al., 1973; CARNEIRO e FREITAS, 1977; COSTA et al., 1979; DUARTE et al., 1982; GUIMARÃES et al., 1983; BIANCHIN, 1996; GUIMARÃES et al., 1996; GIRÃO et al., 1999), os quais classificaram Cooperia spp como a mais prevalente. No entanto, da carga parasitária total, a maior intensidade de infecção apresentada pela Cooperia spp $(77,91 \%)$ em relação ao Haemonchus spp (18,50\%), está semelhante ao encontrado pelos autores supracitados.

Tabela 1 - Prevalência, intensidade média e amplitude total de variação de infecção por helmintos em bovinos na região de Jaboticabal, S P.

\begin{tabular}{|c|c|c|c|c|c|c|}
\hline \multirow{3}{*}{ Helmintos } & \multirow{3}{*}{ Prevalência \% } & \multicolumn{5}{|c|}{ Intensidade de infecção helmíntica } \\
\hline & & $\begin{array}{l}\text { Média entre } \\
\text { parasitados }\end{array}$ & $\begin{array}{l}\text { Média entre } \\
\text { necropsiados }\end{array}$ & $\begin{array}{l}\text { Média entre } \\
\text { necropsiados }\end{array}$ & $\begin{array}{l}\text { Distribuição } \\
\text { percentual }\end{array}$ & \multirow{2}{*}{$\begin{array}{c}\text { Amplitude de } \\
\text { variação }\end{array}$} \\
\hline & & (espécies) & (espécies) & (gênero) & dos gêneros & \\
\hline Bunostomum phlebotomum & 16,66 & 53,71 & 8,95 & 8,95 & 0,083 & $0-110$ \\
\hline Capillaria bovis & 11,90 & 46,00 & 5,48 & 5,48 & 0,279 & $0-140$ \\
\hline Cooperia spatulata & 14,29 & 769,67 & 109,95 & & & $0-2353$ \\
\hline Cooperia punctata & 92,86 & 8733,26 & 8109,45 & 8418,05 & 77,915 & $0-80963$ \\
\hline Cooperia pectinata & 19,05 & 1042,88 & 198,64 & & & $0-3460$ \\
\hline Dictyocaulus viviparus & 16,66 & 21,71 & 3,62 & 3,62 & 0,033 & $0-81$ \\
\hline Eurytrema coelomaticum & 4,76 & 45,00 & 2,14 & 2,14 & 0,020 & $0-70$ \\
\hline Haemonchus placei & 97,62 & 2009,66 & 1961,81 & & & $0-8990$ \\
\hline Haemonchus simillis & 21,43 & 174,78 & 37,45 & 1999,26 & 18,505 & $0-710$ \\
\hline Moniezia benedeni & 4,76 & 1,00 & 0,05 & 0,05 & 0,000 & $0-1$ \\
\hline Oesophagostomum radiatum & 73,81 & 294,52 & 217,38 & 217,38 & 2,012 & $0-1670$ \\
\hline Ostertagia ostertagi & 7,14 & 12,00 & 0,86 & & & $0-29$ \\
\hline Ostertagia lyrata & 4,76 & 3,50 & 0,17 & 1,02 & 0,009 & $0-6$ \\
\hline Strongyloides papillosus & 2,38 & 10,00 & 0,24 & 0,24 & 0,002 & $0-10$ \\
\hline Trichostrongylus axei & 26,19 & 466,00 & 122,05 & & & $0-3880$ \\
\hline Trichostrongylus colubriformis & 2,38 & 20,00 & 0,48 & 122,52 & 1,134 & $0-20$ \\
\hline Trichuris discolor & 38,10 & 66,88 & 25,48 & 25,48 & 0,236 & $0-450$ \\
\hline TOTAL & - & 13770,57 & 10804,20 & 10804,19 & 100,00 & - \\
\hline
\end{tabular}


Utilizando os parâmetros descritos por Skerman e Hillard (1966), os quais classificam a carga parasitária de formas adultas em função das variações de Haemonchus spp em: leve (1 a 400), moderada (400 a $1000)$, grave (1000) e letal (5000), verifica-se que foi encontrado um grande número de bovinos que se enquadram com cargas parasitárias graves e letais desse parasito (Tabela 2).

Observou-seque a prevalência de Oesophagostomum radiatum foi elevada $(73,81 \%)$, com intensidade média de infecção de 217,38 , porém, inferior em relação à Cooperia spp e ao Haemonchus spp. Esses resultados são semelhantes quando comparados a outros trabaIhos realizados no Estado de São Paulo (NOGUEIRA et al., 1976; OLIVEIRA \& MATSUMOTO, 1985; OLIVEIRA, 1988). Landim et al. (2001) apontaram este nematódeo do intestino grosso como o segundo mais prevalente na região nordeste do Estado de São Paulo, da mesma forma como Melo e Ribeiro (1977) no Estado do Mato Grosso do Sul.

A espécie Trichuris discolor, que não havia sido diagnosticada por Nogueira et al. (1976) em Guaíra, SP, apresentou $38,10 \%$ de prevalência. Este resultado está acima do que foi encontrado por Oliveira e Matsumoto (1985), na região de São Carlos, SP; por Duarte et al. (1982), em Cantagalo, RJ e por Carneiro e Freitas (1977), em Goiás; e abaixo da prevalência $(79,31 \%)$ encontrada em Minas Gerais por Costa et al. (1970)

O gênero Trichostrongylus spp. representou apenas $1,134 \%$ da carga parasitária total e suas espécies, $T$. axei e $T$. colubriformis, apresentaram prevalência de $26,19 \%$ e $2,38 \%$, respectivamente. Este resultado confirma os encontrados por Carneiro e Freitas (1977), Costa et al. (1979), Duarte et al. (1982), Oliveira e Matsumoto (1985). Segundo Roberts et al. (1952) e Lee et al. (1960), as temperaturas elevadas influenciam negativamente os estados pré-infectantes dessas espécies. Fato comprovado por Santos et al. (1994), que encontraram o Trichostrongylus spp como gênero de maior prevalência no município de São Francisco de Paula, RS, que é uma região fria.

Por esse mesmo motivo, Ostertagia ostertagi e a O. lyrata, apresentaram baixas prevalências $(7,14 \% \mathrm{e}$ $4,76 \%$, respectivamente) e médias de intensidade de infecção $(12$ e 3,5).

As baixas prevalências de $S$. papillosus (2,38\%) e B. phlebotomum $(16,66)$ podem estar associadas à faixa etária dos bovinos necropsiados, entre 8 e 14 meses, uma vez que, a partir dos cinco meses de idade, os animais começam a apresentar resistência a essas espécies (ROBERTS et al., 1952; VEGORS, 1954; COSTA et al., 1979).

A espécie Dictyocaulus viviparus apresentou médias de infecção entre os animais necropsiados de 3,62\% e entre os parasitados de $21,71 \%$. Esses baixos índices estão de acordo com a afirmação de Honer (1991) de que esta espécie ocorre em todo país, freqüentemente em intensidades baixas de infecção.

Pela análise da Tabela 3, conclui-se que 71,43\% dos bovinos necropsiados encontravam-se parasitados por três a cinco espécies de helmintos. Apenas um animal (2,38\%) apresentou maior diversidade da fauna parasitária, sendo encontradas 10 espécies diferentes de helmintos em seu trato digestivo.

Tabela 2 - Distribuição das infecções por helmintos em intervalo de classe, em bovinos da região de Jaboticabal,SP.

№ de Bezerros / Espécies de Helmintos

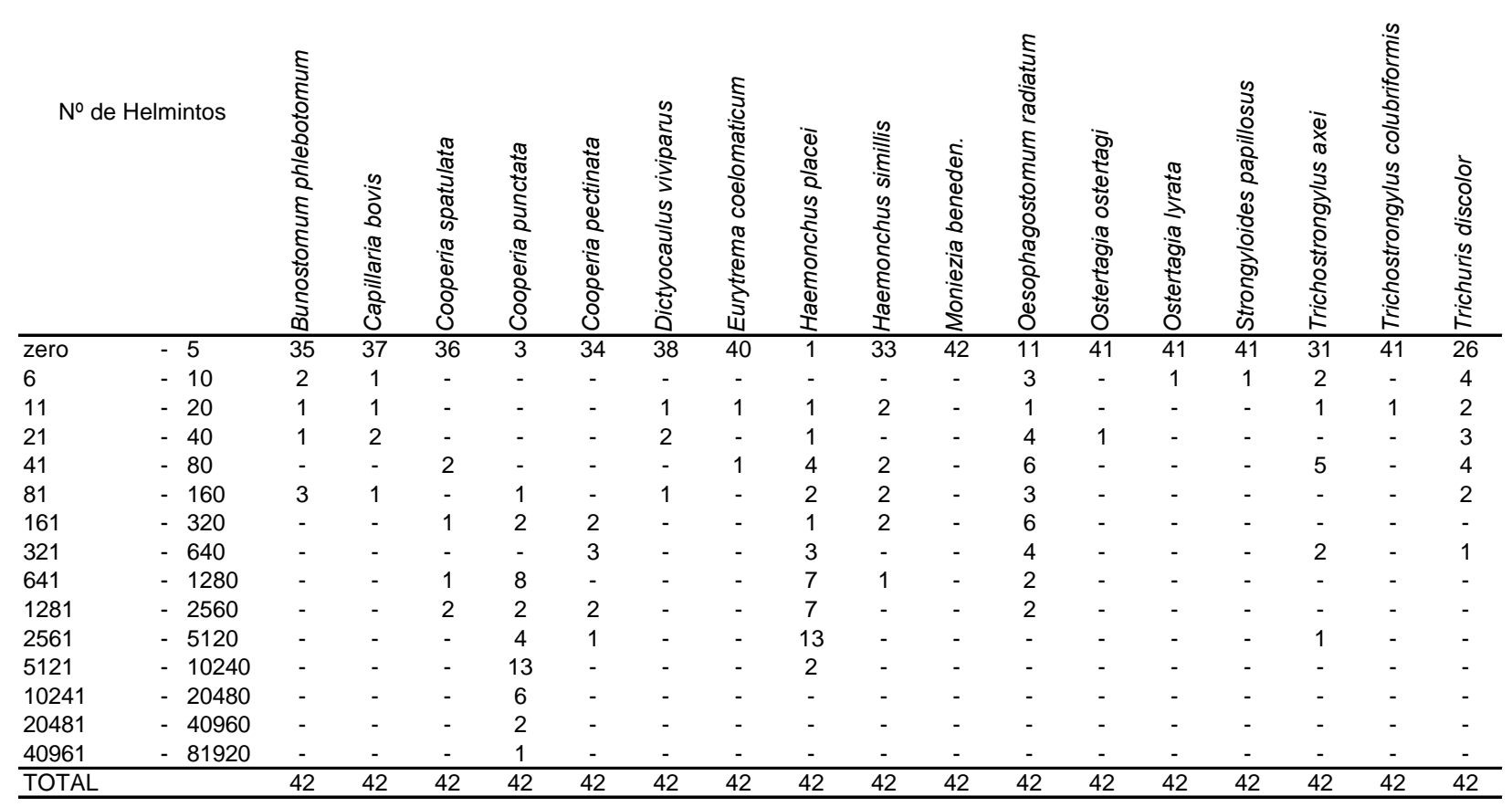


Tabela 3 - Distribuição percentual dos casos de infecções registradas de acordo com o número de espécies de helmintos presentes, em um mesmo hospedeiro. Em bovinos na região de Jaboticabal, SP.

\begin{tabular}{|c|c|c|}
\hline \multirow{2}{*}{$\mathrm{N}^{0}$ de Espécies } & \multicolumn{2}{|c|}{ Bovinos Infectados } \\
\hline & Quantidade & Percentagem \\
\hline 1 & 0 & 0,00 \\
\hline 2 & 4 & 9,52 \\
\hline 3 & 6 & 14,29 \\
\hline 4 & 13 & 30,95 \\
\hline 5 & 11 & 26,19 \\
\hline 6 & 2 & 4,76 \\
\hline 7 & 4 & 9,52 \\
\hline 8 & 1 & 2,38 \\
\hline 9 & 0 & 0,00 \\
\hline 10 & 1 & 2,38 \\
\hline 11 & 0 & 0,00 \\
\hline 12 & 0 & 0,00 \\
\hline 13 & 0 & 0,00 \\
\hline 14 & 0 & 0,00 \\
\hline 15 & 0 & 0,00 \\
\hline 16 & 0 & 0,00 \\
\hline 17 & 0 & 0,00 \\
\hline TOTAL & 42 & 100 \\
\hline
\end{tabular}

\section{Referências Bibliográficas}

ATHAYDE, A.C.R.; COSTA, M.A.; DANTAS, A.M. Freqüência de helmintos em bovinos jovens na região do brejo paraibano. In: SEMINÁRIO BRASILEIRO DE PARASITOLOGIA VETERINÁRIA, 11, 1999, Salvador, Bahia, Brasil. Anais..., Salvador: Colégio Brasileiro de Parasitologia Veterinária, 1999a. p. 146.

ATHAYDE, A.C.R.; COSTA, M.A.; DANTAS, A.M. Freqüência de helmintos em bovinos jovens na região do semi-árido paraibano. In: SEMINÁRIO BRASILEIRO DE PARASITOLOGIA VETERINÁRIA, 11, 1999, Salvador, Bahia, Brasil. Anais..., Salvador: Colégio Brasileiro de Parasitologia Veterinária, 1999b. p. 146.

BIANCHIN, I. Verminose bovina: ocorrência e controle estratégico. In: SIMPÓSIO SOBRE CONTROLE DE PARASITOS, 1., 1996, Campinas. Anais... Campinas: CATI, 1996, p. 41-45.

BIANCHIN, I. et al.. Epidemiologia dos nematódeos gastrintestinais em bovinos de corte nos cerrados e o controle estratégico no Brasil. Campo Grande: EMBRAPA CNPGC, 1996. (Circular Técnica, 24).

CARNEIRO, J.R.; FREITAS, M.G. Curso natural de infecções helmínticas gastrintestinais em bezerros nascidos durante a estação chuvosa em Goiás. Arq. Esc. Vet. U.F.M.G., Belo Horizonte, v. 29, n. 1, p. 49-51, 1977.

CATTO, J.B.; UENO, H. Nematodioses gastrintestinais em bezerros zebu do Pantanal matogrossense. I-Prevalência, intensidade de infecção e variação estacional. Pesq. Agropec. Bras., Brasílav. 16, n. 1, p. 129-140, 1981.

COSTA, A.J. Diagnóstico laboratorial em Parasitologia: I. Helmintologia. Jaboticabal: FCAV-UNESP, 1982. 89p.
COSTA, A.J. et al.. Helmintos parasitos de bezerros do município de Uruana - Goiás, Brasil. Arq. Esc. Vet. UFMG, Belo Horizonte, v. 31, n. 1, p. 33-36, 1979.

COSTA, H.M.A.; FREITAS, M.G.; GUIMARÃES, M.P. Prevalência e intensidade de infestação por helmintos de bovinos procedentes da área de Três Corações. Arq. Esc. Vet UFMG., Belo Horizonte, v. 22, p. 95-101, 1970.

COSTA, H.M.A. et al.. Helmintos parasitos de bezerros na bacia leiteira de Calciolândia-Brasil. Arq. Esc. Vet. UFMG, Belo Horizonte , v. 25, n.2, 1973.

DUARTE, M.J.F.; GOMES, P.A.C.; SANT'ANNA, D.B. Prevalência e intensidade de infecção helmíntica em bezerros de Cantagalo, RJ, Brasil. Pesq. Agropec. Bras., Brasília, v. 17, n. 10, p. 1521-1524, 1982.

FURLONG, J. et al.. Análise bioeconômica do uso de antihelmíntico em bezerros na Zona da Mata de Minas Gerais. Rev. Bras. Parasitol. Vet., São Paulo, v.2, n. 2, p. 119-126, 1993.

GIRÃO, E.S. et al. Verminose bovina. Teresina: EMBRAPA Meio-Norte, 1999. 30p. (Documentos, 41).

GORDON, H.M.; WHITLOCK, H.V. A new techinique for counting nematode eggs in sheep faeces. J. Counn. Sci. Ind. Res. Aust., v. 12, p. 50-52. 1939.

GRISI, L.; NUERNBERG, S. Incidência de nematódeos gastro-intestinais de bovinos, no Estado de Mato - Grosso. Pesq. Agropec. Bras., Sér. Vet., Rio de Janeiro, v. 6, p. 145149, 1971.

GUIMARÃES, M.P. et al.. Gastrintestinal nematode infeccion in beef cattle from the savannah region ("cerrado") of Brazil. Arq. Bras. Med. Vet. Zoot., Belo Horizonte, v. 35, n. 6, p. 845- 
851, 1983.

GUIMARÃES, M.P.; LIMA, P.A.S.; LIMA, W.S. Epidemiologia e controle das helmintoses gastrintestinais de bovinos da região de Teofilo Otoni - Minas Gerais. In: SIMPÓSIO SOBRE CONTROLE DE PARASITOS,1., 1996, Campinas. Anais... Campinas: CATI, 1996, v. 1, n. 0, 1996.

HONER, M.R. Relatório da I reunião sobre epidemiologia de nematódeos no Brasil. Ver. Bras. Parasitol. Vet., São Paulo, v.1, n. 0, p. 5-7, 1991.

JACOBS, D.E.; ARAKAWA, C.H.; GEMELL, M.A.; Mc CALL, J.W.; MYER, G.H.; VANPARIJS, O. World Association for the Advancement of Veterinary Paasitology (W.A.A.V.P.): guidelines for evaluating the efficacy of anthelmintics for dogs and cats. Vet. Parasitol., Amsterdam, v. 52, p. 179202, 1994

LANDIM, V.J.C.; COSTA,A.J.; COSTA, G.H.N.; ROCHA, U.F.; BARBOSA, O.F.; MORAES, F.R. Parasitic nematodes in weaned calves from the northe-east region of São Paulo State, Brazil. ARS Veterinária, Jaboticabal, v. 17, n. 1,p. 4250, 2001

LEE, R.P.; ARMOUR, J.; ROSS, J.G. The seasonal variations of strongyle in Nigerian Zebu Cattle. Brit. Vet. J.,London, v. 116, n.1, p. 34-46, 1960.

LEVINE, N.D. Nematode parasites of domestic animals and of man. Minneapolis: Burgess, 1968. 600p.

MARGOLIS, L.; ESCH, G.W.; HOLMES, J.C.; KURIS, A.M.; SCHAD, G.A. The use of ecological terms in parasitology (report of na ad hoc committee of the american society of parasitologists). J. Parasitol., Lancaster, v. 68, n. 1, p. 131133, 1982.

MELO, H.J.H. ; RIBEIRO, H.S. Helmintos parasitas dos animais domésticos no Estado do Mato-Grosso. Arq. Esc. Vet. U.F.M.G., Belo Horizonte, v. 29, n. 2, p. 161-164, 1977.

NOGUEIRA, C.Z.; COSTA, A.J.; MACHADO,R.Z.; KASAI, N. Evolução natural das infecções por nematóides parasitos gastrintestinais de bezerros nascidos durante a estação chuvosa em Guaíra, Estado de São Paulo, Brasil. Científica, São Paulo, v. 4, n. 3, p. 346-355, 1976.

OLIVEIRA, G.P. Epidemiologia dos nematódeos gastrintestinais de bovinos leiteiros no município de São Carlos, Estado de São Paulo. Pesq. Agropec. Bras., Brasília, v. 23, n. 2, p. 189-195, 1988.

OLIVEIRA, G.P. ; MATSUMOTO, T. Prevalência e intensidade de infecção por helmintos em bovinos da bacia leiteira de São Carlos, São Paulo. Pesq. Agropec. Bras., Brasília, v. 20, n. 12, p. 1415-1418, 1985.

PINHEIRO, A. C.; BORBA, M.F.S.; CORRÊA, M.B.C.; ALVESBRANCO, F.P.J. Prevalência e intensidade das infecções helmínticas em bezerros de raça de corte nascidos na primavera e outono, em Bagé/RS. In: SIMPÓSIO SOBRE CONTROLE DE PARASITOS, 1., 1996, Campinas. Anais... Campinas: CATI, 1996. v. 1.

RAMOS , C.I. Epidemiologia das helmintoses gastrintestinais de bovinos de corte no planalto catarinense. Dinâmica populacional de Cooperia spp. e Trichostrongylus spp. 1983. 104f. Dissertação (Mestrado) - Universidade Federal do Rio Grande do Sul, Porto Alegre, 1983.

RASSIER, D.S.S. Prevalência e variação estacional de nematódeos parasitas gastrintestinais em bovinos na encosta sudeste do Rio Grande do Sul. 1975. Dissertação
(Mestrado) - Universidade Federal de Minas Gerais, Belo Horizonte, 1975.

ROBERTS, F.H.S.; O'SULLIVAN, P.J.; RIEK, R.F. The epidemiology of parasitic gastro-enteritis of cattle. Aust. J. Agric. Res., Victoria v. 3, p. 187-226, 1952.

SANTANA, J.C.R.; BARBOSA, S.J.; NETO, R.B.; DALUZ, E.A.T. Ocorrência e variação estacional de helmintos parasitos de bovinos na região de Itaju do Colômbia. Pesq. Agropec. Bras., Brasília, v. 24, n.10, p. 1235-1240, 1989.

SANTOS, I.C.S.; CORREA, B.L.; SCAINI, C.J.; CARNIEL, R.L. Verminose bovina no distrito de Lajeado Grande, São Francisco de Paula - RS. Lav. Arrozeira, Porto Alegre, v. 47, n. 415, p. 6-14, 1994.

SKERMAN, K.D. ; HILLARD, J.J. A handbook for studies of helminth parasites of ruminants. Beirut: Near East Animal Helth Institutes, 1966.

VEGORS, H.H. Experimental infection of calves with Strongyloides papillosus(nematoda). Amer. J. Vet. Res., Chicago, v. 15, p. 429-433, 1954.

WHITLOCK, H.V. Some modifications of the McMaster helminth egg-couting technique and apparatus. J. Counm Sci. Ind. Res., v.21, p.177-180, 1948. 